\title{
Multi-objective Genetic Approach for Analog Circuit Sizing using SVM Macro-model
}

\author{
D. Boolchandani \\ Dept. of ECE \\ NIT Jaipur, India - 302017 \\ Email: dbool@ieee.org
}

\author{
Anupam Kumar \\ Centre for Development of Telematics \\ Mehrauli Road, New Delhi, India - 110016 \\ Email: anupam.mnit@yahoo.co.in
}

\author{
Vineet Sahula \\ Dept. of ECE \\ NIT Jaipur, India - 302017 \\ Email: sahula@ieee.org
}

\begin{abstract}
Analog Circuit sizing is the task to determine the sizes of all components in the circuit during automated synthesis. Randomized combinatorial optimization algorithms are desired for quicker determination of a set of optimal sizes of the components. These algorithms require set of multiple performance parameters, for a very large number of sized circuits. Therefore the reduction in time required to estimate these performance parameters is also highly desired. For the purpose of estimation of performance parameters, we employ Support Vector Machine (SVM) based macro-models of analog circuits, instead of using SPICE simulation. These SVM macro-models are not only faster to evaluate, but use of efficient kernel functions has also made them almost as accurate as SPICE. In this paper, we report multi-objective genetic algorithm for simultaneous optimization of multiple performance parameters. We compute the Pareto optimal points for various performance parameters of a twostage op-amp circuit in $180 \mathrm{~nm}$ technology. We perform SVM classification and regression using Least Square SVM toolbox [1] with MATLAB. HSPICE was used to generate data-set from simulation of two-stage op-amp, which was used to train the SVM macro-model. The results pertaining to total time consumed in sizing task are very encouraging. We observed 'time taken' in one evaluation by SVM macromodel as compared to HSPICE is upto two order smaller, resulting in speed-up of approximately 20.
\end{abstract}

\section{INTRODUCTION}

Circuit synthesis of analog circuit is the task to determine the size of all components in the circuit so that it is able to meet the set of performance specifications. Optimum sizes of the components are determined using stochastic combinatorial optimization method such as simulated annealing and genetic algorithms. Since, performance parameters for great number of circuit sizing values are needed by these algorithms, the reduction in time is highly desired to estimate these performances.

Many macromodeling techniques have been proposed to match the non-linear performance functions to design parameters [2]-[4]. In this paper, we have used Support Vector Machine (SVM) [5]-[7] based macro-models to provide robust and accurate estimate of performance parameters for two stage op amps. The utility of these models is demonstrated in circuit sizing methodology using multi-objective genetic algorithm optimization. The SVM models used in this work were trained using data generated directly from SPICE and therefore are able to provide SPICE level accuracy. Since, the evaluation

This research work was supported by grant from Ministry of Communication \& IT, Government of India under the sponsored project SMDP-VLSI-2. time taken by the SVM models is much less than the time needed for a full SPICE simulation, the models can be used inside an optimization loop as cost function during circuit synthesis.

Performance Macromodeling usually consist of two steps: feasibility design space identification and performance macromodels generation. A feasibility design space is defined as a multidimensional space in which every design satisfies all the design constraints. The minimum set of constraints is the one that ensures the correct functionality of the given circuit topology. Performance macromodels are only constructed and thereby valid in the functionally correct design space. Support vector machines (SVMs) are used as classifier to identify the feasible design space of analog circuits and then as regressor to model performance function of the circuits. Once SVM models are developed for different performance parameters of op-amp, one model for each of the parameter, the op amp can then be synthesized for different performance specifications using general genetic algorithm. However, often a number of parameters in the performance functions compete against each other. Hence, selecting the design variable such that one of the performance parameter is optimal will generally not result in optimal values for other parameters. Thus, the circuit optimization problem turns out to be a multi objective optimization problem. Using multi-objective genetic algorithm, hyper surface of Pareto-optimal design points is calculated that is helpful to designer to make trade-offs between different performance parameters. This multi-objective optimization approach is evaluated while sizing the two stage op amp circuit.

Our contribution lies in using efficient kernel functions for SVM classification as well as regression during circuit sizing. We illustrate the efficacy and applicability of the macromodels to multi-objective circuit sizing of select analog circuits. Support vector machine based classification \& regression are discussed next, in Section II, along with Pareto surface generation method. In Section III, we illustrate experimental setup for single objective as well as multi-objective sizing of two stage op amp. We also report results in Section III. We conclude in Section IV. 


\section{BACKGROUND \& RELATED WORK}

\section{A. SVM Classification}

The classification is a procedure to separate out infeasible design points from feasible ones. In this section, our further discussion is based on [5] [8]. All such points together constitute input data to classifier. We consider each of $\mathrm{N}$ data points $x_{k} \in R^{n}, k=1, \cdots, N$ to be associated with a label $y_{k} \in\{+1,-1\}$ thus classifying the data into one of two sets. In the simplest SVM formulation, the problem of finding a general representation of the classifier $y(x)$ becomes that of the construction of a hyper-plane $\omega^{T} x_{k}+b$ which provides 'maximal separation $\frac{2}{\|\omega\|^{2}}$ between points $x_{k}$ belonging to the two classes. This give rise to an optimization problem of the form given by (1), and its dual in (2) using Lagrangian form. Here the $\frac{1}{2} \omega^{T} \omega$ term represents a cost function to be minimized in order to maximize separation. The constraints are formulated such that the nearest points $x_{k}$ with labels [either +1 or -1 ] are (with appropriate input space scaling) at least $\frac{1}{\|\omega\|^{2}}$ distant, from the separating hyper-plane.

$$
\begin{aligned}
& P: \min _{\omega, b} \quad \frac{1}{2} \omega^{T} \omega \quad \text { s.t. } \quad y_{k}\left[\omega^{T} x_{k}+b\right] \geq 1 \\
& D: \max _{\alpha} \mathcal{L}(w, b ; \alpha)
\end{aligned}
$$

Here, $\mathcal{L}(\omega, b ; \alpha)=\frac{1}{2} \omega^{T} \omega-\sum_{k=1}^{N} \alpha_{k}\left(y_{k}\left[\omega^{T} x_{k}+b\right]-1\right)$ and $\alpha_{k}$ are the Lagrange multipliers. After applying the conditions as in (3) for optimality, kernel trick [7] and eliminating $\omega$ by expressing it in terms of $\alpha=\left[\alpha_{1}, \ldots, \alpha_{N}\right]$, we arrive at a Quadratic Programming (QP) problem as in (4).

$$
\begin{aligned}
& \frac{\partial \mathcal{L}}{\partial \omega}=0, \frac{\partial \mathcal{L}}{\partial b}=0, \frac{\partial \mathcal{L}}{\partial \alpha_{k}}=0, \\
& \min (\alpha Q \alpha+B \alpha),
\end{aligned}
$$

for suitably defined matrices $Q, B$. Having solved for $\alpha$, the following classifier representation is obtained

$$
y(x)=\operatorname{sign}\left[\sum_{k=1}^{\# S V} \alpha_{k} y_{k} x_{k}^{T} x+b\right] .
$$

Here \#SV represents the number of non-zero Lagrange multipliers $\alpha_{k}$, called support vectors, corresponding to input data $x_{k}$. A key feature of the Support Vector Machines is the ability to replace the input data by a non-linear function $\phi(x)$ operating on the input data. This may be viewed as mapping the input data to higher dimensional space, to enable classification of data that is not linearly separable in the original input space. To do this, we formally replace $x_{k}^{T} x$ (the dot product between a support vector $x_{k}$ and any point $x$ of the input space) in (5) by $\phi\left(x_{k}\right)^{T} \phi(x)$ to represent the action of this mapping, obtaining

$$
y(x)=\operatorname{sign}\left[\sum_{k=1}^{\# S V} \alpha_{k} y_{k} \phi\left(x_{k}\right)^{T} \phi(x)+b\right] .
$$

The expression $\phi\left(x_{k}\right)^{T} \phi(x)$ may under certain conditions be replaced by a Kernel function $K\left(x_{k}, x\right)$. There are different kernel functions that provide the SVM, the ability to model complicated separation hyperplanes. We use the efficient kernels proposed in [9] to built SVM model for classifying feasible design space.

\section{B. SVM Regression}

In the case of function regression, the labels $y_{k} \in\{-1,+1\}$ represented by the $\{+1,-1\}$ valued function $y(x)$ are replaced by the real valued $y_{k} \in R$. Further by a certain nonlinear mapping $\phi$, the training pattern $x_{t}$ is mapped into some feature space, in which a real valued function $y(x)$ is defined as follows.

$$
y(x)=\omega^{T} \phi(x)+b \text { with } \omega \in R^{N}, b \in R
$$

Here, $\phi() \quad:. \quad R^{n} \rightarrow R^{n_{h}}$ is the mapping to the high dimensional and potentially infinite dimensional feature space. For the Least-Squares SVM regression error variables for the fitting problem are as given in (8).

$$
e_{k}=w^{T} \phi\left(x_{k}\right)+b-y_{k} k=1, \ldots, N
$$

Given a training set $\left\{x_{k}, y_{k}\right\}_{k=1}^{N}$ following optimization problem is formulated in the primal weight space together with the $\mathrm{N}$ constraints of (8).

$$
P: \min _{w, b, e} J_{p}(w, e)=\quad \frac{1}{2} w^{T} w+\gamma \frac{1}{2} \sum_{k=1}^{N} e_{k}^{2}
$$

This formulation involves the trade off between a cost function term and a sum of squared errors governed by the trade-off parameter $\gamma$. In the regression formalism the term $\frac{1}{2} w^{T} w$ is no longer related to hyper-plane separation, but instead determines the smoothness of the resulting model. In fact, the primal problem in the LS-SVM formalism is wholly equivalent to a ridge regression problem formulated in the feature space, with parameter $\gamma$ performing the role of smoothing parameter. The dual Lagrangian-based formulation is given in (10), where $\mathcal{L}=J_{p}(w, e)-\sum_{k=1}^{N} \alpha_{k}\left\{w^{T} \phi\left(x_{k}\right)+b+e_{k}-y_{k}\right\}$ and $\alpha_{k}$ are Lagrange multipliers.

$$
\begin{aligned}
& D: \max _{\alpha} \mathcal{L}(w, b, e ; \alpha) \\
& \mathcal{L}=J_{p}(w, e)-\sum_{k=1}^{N} \alpha_{k}\left\{w^{T} \phi\left(x_{k}\right)+b+e_{k}-y_{k}\right\}
\end{aligned}
$$

The conditions for optimality are given by (12).

$$
\left\{\begin{array}{l}
\frac{\partial \mathcal{L}}{\partial w}=0 \rightarrow w=\sum_{k=1}^{N} \alpha_{k} \phi\left(x_{k}\right) \\
\frac{\partial \mathcal{L}}{\partial b}=0 \rightarrow \sum_{k=1}^{N} \alpha_{k}=0 \\
\frac{\partial \mathcal{L}}{\partial e_{k}}=0 \rightarrow \alpha_{k}=\gamma e_{k}, k=1, \ldots, N \\
\frac{\partial \mathcal{L}}{\partial \alpha_{k}}=0 \rightarrow w^{T} \phi\left(x_{k}\right)+b+e_{k},-y_{k}=0
\end{array}\right.
$$


After elimination of the variables $w$ and $e$ one gets the solution as in (13), where $y=\left[y_{1} ; \ldots ; y_{N}\right], 1_{v}=[1 ; \ldots ; 1]$ and $\alpha=\left[\alpha_{1} ; \ldots ; \alpha_{N}\right]$. The kernel trick is applied as in (14).

$$
\begin{aligned}
& {\left[\begin{array}{cc}
0 & 1_{N}^{T} \\
1_{N} & \Omega+I / \gamma
\end{array}\right]\left[\begin{array}{c}
b \\
\alpha
\end{array}\right]=\quad\left[\begin{array}{l}
0 \\
y
\end{array}\right]} \\
& \Omega_{k l}=\quad \phi\left(x_{k}\right)^{T} \phi\left(x_{l}\right) \\
& =\quad K\left(x_{k}, x_{l}\right) \quad k, l=1, \ldots, N
\end{aligned}
$$

The resulting LS-SVM model, after elimination of variables and applying kernel trick [7], for function estimation becomes as in (15). The function $K\left(x, x_{k}\right)$ corresponds to a dot product in some feature space.

$$
y(x)=\sum_{k=1}^{N} \alpha_{k} K\left(x, x_{k}\right)+b
$$

\section{Pareto optimal surface}

Concept of Pareto optimality is used to characterize competing objectives. For a problem, where multiple performances need be optimized, let there be vector of objectives $F(x)=$ $\left[F_{1}(x), F_{2}(x), \ldots \ldots, F_{m}(x)\right]$ that must be traded off. Multiobjective optimization is concerned with the minimization of a vector of objectives $F(x)$ that can be the subject of a number of constraints or bounds as given in (16). The performance vector $\mathrm{F}(\mathrm{x})$ maps parameter space into objective function.

$$
\begin{array}{r}
\min _{x \in R^{n}} F(x) \text { subject to } G_{i}(x)=0, \text { for } i=1, \cdots, k_{e} \\
\quad G_{i}(x) \leq 0, i=k_{e+1}, \ldots, k ; \text { and } l \leq x \leq u
\end{array}
$$

\section{Pareto optimal solution}

Let us consider a feasible region, $\Omega$, in the parameter space. $X$ is an element of the n-dimensional real numbers $x \in R^{n}$ that satisfies all the constraints i.e., $\Omega=\left\{x \in R^{n}\right\}$, subject to $G_{i}(x)=0, i=1, \ldots, k_{e}$, and $G_{i}(x) \leq 0, i=k_{e+1}, \ldots, k$, $l \leq x \leq u$. Point $x^{*} \in \Omega$ is a Pareto optimal solution if for some neighborhood of $x^{*}$ there doesn't not exist a $\Delta x$ such that $\left(x^{*}+\Delta x\right) \in \Omega$ and $F_{i}\left(x^{*}+\Delta x\right) \leq F_{i}\left(x^{*}\right), i=1, \ldots, m$, and $F_{j}\left(x^{*}+\Delta x\right) \leq F_{j}\left(x^{*}\right)$ for at least one $\mathrm{j}$. In the two dimensional representation of the Fig. 1, the set of Pareto optimal solution lies on the curve between $\mathrm{C}$ and D. Points $\mathrm{A}$ and $\mathrm{B}$ represent specific Pareto optimal points because an improvement in one objective, $F_{1}$, requires a degradation in the other objective, $F_{2}$, i.e., $F_{1 B}<F_{1 A}, F_{2 B}>F_{2 A}$.

The Pareto curve is the set of $x^{*}$ where there are no other solutions for which simultaneous improvement in all objectives can occur. A general goal in multi-objective optimization is constructing the Pareto optima. We have used GA-multiobjective tool box in MATLAB to create a set of Pareto optima. It uses genetic algorithms to identify Pareto points.

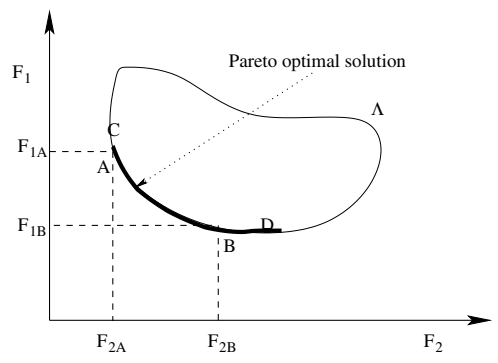

Figure 1. Set of Pareto optimal solutions

\section{EXPERIMENTAL SETUP \& RESULTS}

A known instance of all the design variable is considered a tuple. HSPICE is used on each of these tuples of design variables to evaluate performance and verify functional and performance constraints. For given set of tuples which satisfy both functional and performance constraints, output is taken as '1' otherwise as '-1'. This forms set of input and output data pair. Some of these data pairs are used to train SVM classifier and while the remaining are used for validation i.e. to check the accuracy of classifier.

The circuit simulator HSPICE is also used to compute several standard performance parameters describing the functionality of operational amplifier circuit. By doing SPICE simulation for many combinations of op amp transistor sizes, training and validation data sets are produced. These are then used to generate SVM model for the performance parameter. A general iterative methodology as suggested in [9] and reproduced in Fig. 2, is used for SVM model development.

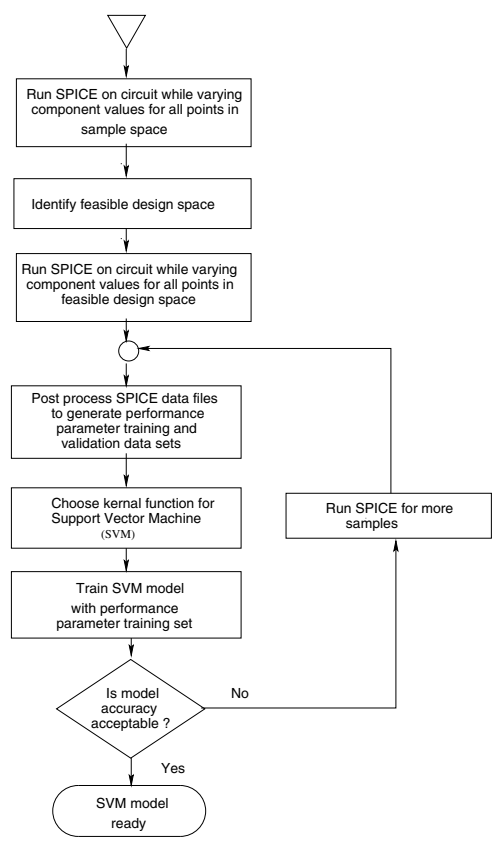

Figure 2. Flow diagram of SVM model development.

After training, the SVM model for given op amp topology is used to provide estimates of op amp performance parameter 
during optimization. Op amp under various performance constraints is then synthesized using a genetic algorithm. Genetic algorithm is chosen since it is robust in the presence of multiple constraints and is insensitive to the nature of the cost function. Genetic algorithm have been successfully used previously for synthesis of analog circuits [10], [11]. The block diagram in Fig. 3 depicts the synthesis methodology using the SVM models.

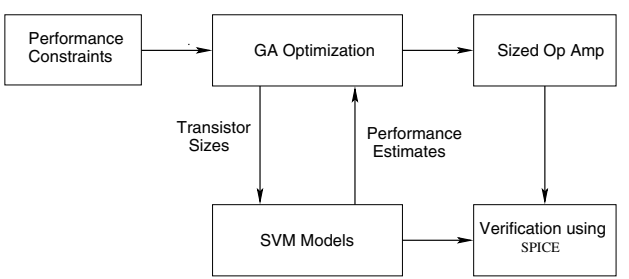

Figure 3. Block diagram of circuit synthesis via a genetic optimization engine with SVM models

\section{A. Nominal Sizing of Two Stage op-amp}

A single output two-stage op-amp is shown in Fig. 4 . The circuit has 8 transistors, compensation and load capacitance and a reference bias current. The length of all transistors are fixed to a $1 \mu \mathrm{m}$. This immediately eliminates nearly half of the free design parameters. Further imposing sizing rule mentioned in [12], results in the 5-dimensional parametric configuration for the two-stage op-amp. The design variables and geometry constraints are shown in Table I. Functional and Performance constraints are shown in Table II. The functional constraints ensure all the transistors are on and in saturation region with some margin. We set $V_{\text {on,min }}$ and $V_{\text {sat,min }}$ to $0.1 \mathrm{~V}$.

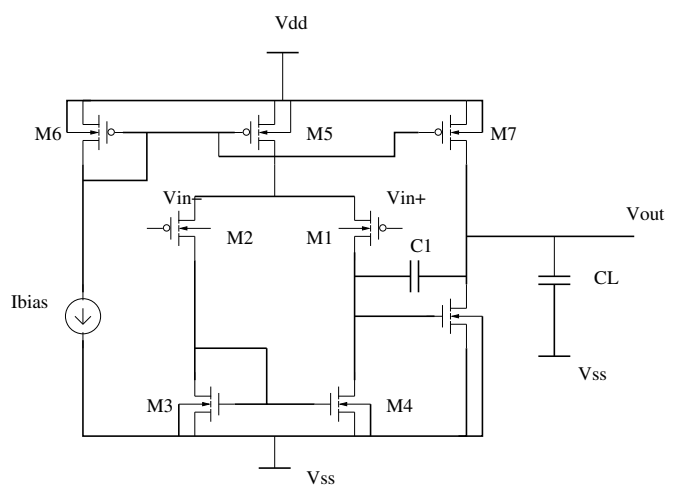

Figure 4. Two stage op amp

Table I

DESIGN VARIABLES For Two STAGE OP AMP

\begin{tabular}{|c|c|}
\hline Design parameters & Geometric constraints \\
\hline \hline$W_{1}=W_{2}$ & {$[1 \mu \mathrm{m}, 100 \mu \mathrm{m}]$} \\
\hline$W_{3}=W_{4}$ & {$[1 \mu \mathrm{m}, 50 \mu \mathrm{m}]$} \\
\hline$W_{5}$ & {$[1 \mu \mathrm{m}, 100 \mu \mathrm{m}]$} \\
\hline$W_{7}$ & {$[1 \mu \mathrm{m}, 100 \mu \mathrm{m}]$} \\
\hline $\mathrm{Cc}$ & {$[5 \mathrm{pF}, 20 \mathrm{pF}$} \\
\hline
\end{tabular}

Table II

DESIGN CONSTRAINTS FOR TWO-STAGE OP-AMP

\begin{tabular}{|c|c|}
\hline $\begin{array}{c}\text { Functional } \\
\text { constraints }\end{array}$ & $V_{g s}-V_{t h} \geq V_{\text {on, min }}$ \\
\hline $\begin{array}{c}\text { Performance } \\
\text { constraints }\end{array}$ & Phase Margin $\geq 45^{\circ}$ \\
\hline
\end{tabular}

Least Square SVM toolbox interfaced with MATLAB was used for developing the classifier to identify feasible design space. Data in feasible design space was used to develop SVM regressor models for different performance parameters like open loop gain, phase margin and unity gain frequency. The respective correlation coefficients between outputs of SVM model and SPICE for various performance parameters of two stage op-amp are computed and illustrated in Fig. 5. The correlation coefficient between outputs of SVM model and SPICE of various performance parameters of two stage opamp are found close to one, which implies that models are quite accurate. Further from the Table IV we see that these models are also quite efficient as the time taken to compute performance parameters is very less as compared to HSPICE.
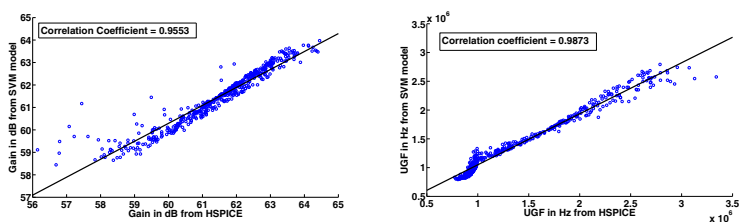

Figure 5. Correlation factors for (a) Gain and (b) UGF

Table III

PERFORMANCE SPECIFICATIONS OF TWO-STAGE OP-AMP

\begin{tabular}{|c|c|}
\hline Performance & specifications \\
\hline \hline Open-loop gain & $\geq 60 \mathrm{~dB}$ \\
\hline Phase-margin & $\geq 70^{\circ}$ \\
\hline UGF & $\geq 2 \mathrm{MHz}$ \\
\hline
\end{tabular}

GA toolbox of MATLAB was used to run genetic algorithm to size the two stage op amp with objective function to minimize area and at same time satisfying performance specifications given in Table II . It was run for 100 generations with population size of 20. Crossover function used was scattered and the mutation function selected was Gaussian. Elite count was taken as 2 and crossover fraction 0.8. The dimensions of transistors, compensation capacitor and bias current of two stage op amp optimized for minimum area and constraints, obtained through genetic optimization algorithm are shown in

Table IV

COMPARISON OF TIME TAKEN TO EVALUATE PERFORMANCE PARAMETERS OF TWO STAGE OP AMP

\begin{tabular}{|c|c|c|c|}
\hline Performance parameter & SVM-Model & HSPICE & Speed-up \\
\hline Open loop Gain & $0.4449 \mathrm{Sec}$ & $10.0 \mathrm{Sec}$ & 22.48 \\
\hline Unity Gain Frequency & $0.4450 \mathrm{Sec}$ & $10.0 \mathrm{Sec}$ & 22.47 \\
\hline Phase Margin & $0.4457 \mathrm{Sec}$ & $10.0 \mathrm{Sec}$ & 22.44 \\
\hline
\end{tabular}


Table V

DESIGN VARIABLES RESULTING FROM OPTIMAL SIZING OF TWO STAGE OPAMP

\begin{tabular}{|c|c|}
\hline $\begin{array}{c}\text { Design } \\
\text { Variable }\end{array}$ & $\begin{array}{c}\text { Optimal } \\
\text { Size }\end{array}$ \\
\hline$W_{1}=W_{2}$ & $6.48 \times 10^{-05} \mu \mathrm{m}$ \\
\hline$W_{3}=W_{4}$ & $9.63 \times 10^{-06} \mu \mathrm{m}$ \\
\hline$W_{5}$ & $2.87 \times 10^{-05} \mu \mathrm{m}$ \\
\hline$W_{7}$ & $6.50 \times 10^{-05} \mu \mathrm{m}$ \\
\hline $\mathrm{Cc}$ & $6.79 \times 10^{-12} \mathrm{~F}$ \\
\hline
\end{tabular}

Table VI

PERFORMANCE PARAMETERS RESULTING FROM OPTIMAL SIZING OF TWO STAGE OPAMP

\begin{tabular}{|c|c|c|}
\hline $\begin{array}{c}\text { Performance } \\
\text { Parameters }\end{array}$ & HSPICE & $\begin{array}{c}\text { SVM } \\
\text { Model }\end{array}$ \\
\hline Open-loop gain & $61.71 \mathrm{~dB}$ & $61.23 \mathrm{~dB}$ \\
\hline Phase-margin & $122^{\circ}$ & $133^{\circ}$ \\
\hline UGF & $2.75 \mathrm{MHz}$ & $3.05 \mathrm{MHz}$ \\
\hline
\end{tabular}

Table V . Values of performance parameter obtained through HSPICE as well as SVM models is also shown in Table VI . All the performance parameter satisfy the constraint specified in sizing algorithm. Computation time for sizing turned out to be 277.1 seconds.

\section{B. Sizing of Cascode Op amp}

The circuit of cascode op-amp is shown in Fig. 6. We fix the lengths of all transistors to $1 \mu \mathrm{m}$. Imposing sizing rules [12] similar to that of two-stage op-amp we get five design variables for cascode op-amp. Load capacitance is set to $1 \mathrm{pf}$. The design variables and geometry constraints are shown in Table VII. Other constraints shown in Table VIII. The functional constraints ensure all the transistors are on and in saturation region with some margin. We set $V_{o n, \text { min }}$ and $V_{\text {sat,min }}$ to $0.1 \mathrm{~V}$. Similar to two stage op amp SVM models were built for different performance parameters like phase margin, unity gain frequency, CMRR, PSRR and slew rate.

Cascode op amp is synthesized for minimum area and constraints given in Table VIII. The dimensions of transistors, compensation capacitor and bias current of cascode op amp obtained through genetic optimization algorithm are shown in Table X . Values of performance parameter obtained through HSPICE as well as SVM models is also shown in Table XI .

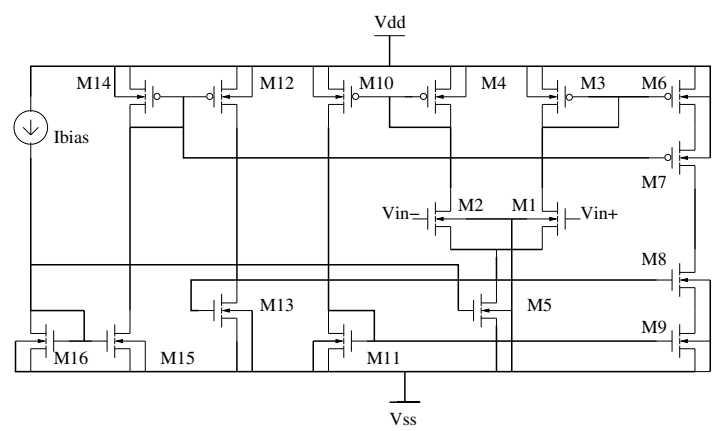

Figure 6. Cascode Op amp
Table VII

DESIGN VARIABLES FOR CASCODE OP-AMP

\begin{tabular}{|c|c|}
\hline Design parameters & Geometric constraints \\
\hline \hline$W_{1}=W_{2}$ & {$[1 \mu \mathrm{m}, 100 \mu \mathrm{m}]$} \\
\hline$W_{3}=W_{4}$ & {$[1 \mu \mathrm{m}, 100 \mu \mathrm{m}]$} \\
\hline$W_{5}=W_{6}$ & {$[1 \mu \mathrm{m}, 100 \mu \mathrm{m}]$} \\
\hline Ibias & {$[2 \mu \mathrm{A}, 20 \mu \mathrm{A}]$} \\
\hline$C_{L}$ & {$[1 p F, 10 p F]$} \\
\hline
\end{tabular}

Table VIII

DESIGN CONSTRAINTS FOR CASCODE OP-AMP

\begin{tabular}{|c|c|}
\hline Functional & $V_{g s}-V_{t h} \geq V_{o n, \min }$ \\
\hline \hline constraints & $V_{d s} \geq V_{g s}-V_{t h}+V_{\text {sat }, \text { min }}$ \\
\hline $\begin{array}{r}\text { Performance } \\
\text { constraints }\end{array}$ & Phase Margin $\geq 60^{\circ}$ \\
\hline
\end{tabular}

All the performance parameter satisfy the constraint specified in sizing algorithm.

\section{Multi-objective Sizing of op amp}

Using multi-objective genetic optimization [13], the surface of Pareto-optimal design point is computed for three performance parameters of two stage op amp. Performance parameters that are considered are open-loop gain, phasemargin and unity gain frequency. Multivariate regression model is developed using SVM of two stage op amp for the above three output performance function of op amp. The multivariate model has SPICE level accuracy as it is trained by data obtained through SPICE simulation and is quite fast in evaluation of circuit performances for given set of design variables. This models is then used within multi-objective genetic algorithm to generate set of Pareto-optimal points. Pareto optimal points obtained for two stage op amp are shown in Table XII. It was run for 122 generations with population size of 60 . Crossover function used was scattered with crossover fraction of 0.8 . Pareto-optimal plots for two stage op amp are shown in Fig. 7 and Fig. 8. In the two figures, we observe that there is an obvious trade off between gain and phase margin and also between gain and unity gain frequency. Pareto-optimal points

Table IX

PERFORMANCE CONSTRAINTS FOR CASCODE OP AMP

\begin{tabular}{|c|c|}
\hline Performance & Constraints \\
\hline \hline CMRR & $\geq 100 \mathrm{~dB}$ \\
\hline PSRR & $\geq 120 \mathrm{~dB}$ \\
\hline Phase-margin & $\geq 70^{\circ}$ \\
\hline Slew rate & $\geq 1.25 \times 10^{06} \mathrm{~V} / \mathrm{sec}$ \\
\hline Unity-gain frequency (UGF) & $\geq 1 \times 10^{06} \mathrm{~Hz}$ \\
\hline
\end{tabular}

Table X

DESIGN VARIABLES OBTAINED FROM OPTIMAL SIZING OF CASCODE OP AMP

\begin{tabular}{|c|c|}
\hline Design Variable & Optimal Size \\
\hline$W_{1}=W_{2}$ & $2.60 \times 10^{-05} \mu \mathrm{m}$ \\
\hline$W_{3}=W_{4}$ & $9.84 \times 10^{-06} \mu \mathrm{m}$ \\
\hline$W_{5}=W_{6}$ & $7.87 \times 10^{-05} \mu \mathrm{m}$ \\
\hline Ibias & $1.72 \times 10^{-05} \mu \mathrm{A}$ \\
\hline$C_{L}$ & $3.70 \mathrm{pF}$ \\
\hline
\end{tabular}


Table XI

PERFORMANCE PARAMETERS OBTAINED FROM OPTIMAL SIZING OF CASCODE OP AMP

\begin{tabular}{|c|c|c|}
\hline Performance Parameter & HSPICE & SVM-Model \\
\hline CMRR & $143 d B$ & $139 \mathrm{~dB}$ \\
\hline Phase-margin & $71^{\circ}$ & $70^{\circ}$ \\
\hline PSRR & $151 \mathrm{~dB}$ & $136 \mathrm{~dB}$ \\
\hline Slew rate & $1.76 e 06$ & $1.43 \times 10^{06} \mathrm{~V} / \mathrm{sec}$ \\
\hline UGF & $1.48 e 06$ & $1.29 \times 10^{06} \mathrm{~Hz}$ \\
\hline
\end{tabular}

Table XII

PARETO-OPTIMAL VALUES OF PERFORMANCE PARAMETERS FOR TWO STAGE OP AMP

\begin{tabular}{|c|c|c|}
\hline $\begin{array}{c}\text { Open-loop gain } \\
\text { (in } \mathrm{dB} \text { ) }\end{array}$ & $\begin{array}{c}\text { Phase-margin } \\
\text { (in degrees) }\end{array}$ & $\begin{array}{c}\text { Unity-gain frequency } \\
\text { (in MHz) }\end{array}$ \\
\hline 61.86 & 145 & 3.22 \\
\hline 64.30 & 84 & 0.91 \\
\hline 61.90 & 149 & 3.20 \\
\hline 63.62 & 88 & 1.44 \\
\hline 63.35 & 91 & 1.78 \\
\hline 62.36 & 123 & 2.70 \\
\hline 63.97 & 83 & 1.06 \\
\hline 62.78 & 114 & 2.32 \\
\hline 62.17 & 135 & 2.95 \\
\hline 63.19 & 102 & 1.99 \\
\hline 61.92 & 144 & 3.20 \\
\hline 63.66 & 86 & 1.34 \\
\hline 62.57 & 119 & 2.47 \\
\hline 62.21 & 129 & 2.87 \\
\hline 62.98 & 108 & 2.15 \\
\hline 61.85 & 145 & 3.21 \\
\hline 63.04 & 97 & 2.08 \\
\hline
\end{tabular}

of three performance parameters of two stage op amp is shown in Fig. 9. Total computation time taken by GA-multi-objective algorithm was 437.63 seconds.

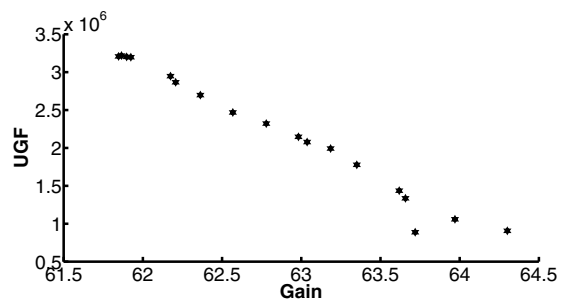

Figure 7. Pareto optimal front for UGF with Gain

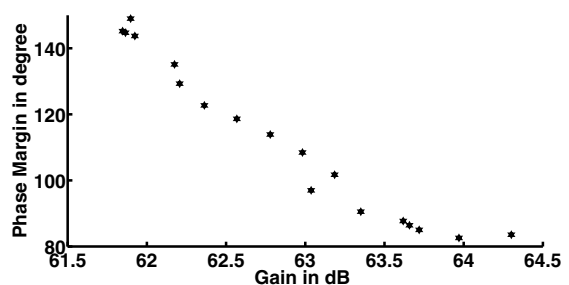

Figure 8. Pareto optimal fronts for Gain with Phase margin

\section{CONCLUSIONS}

We have proposed multi-objective genetic algorithm formulation based on SVM macro models for analog circuit

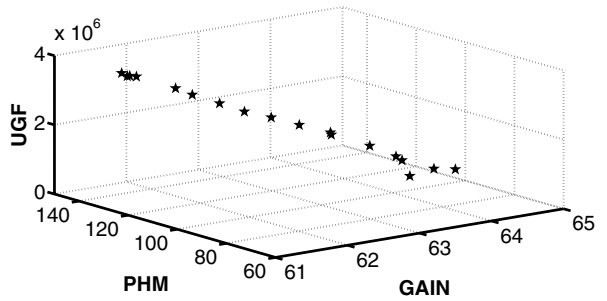

Figure 9. Pareto points on surface for three performance parameters

sizing. The analog macro-models replace Spice simulators, used for circuit fitness evaluation inside GA, consuming a very little time and are almost as accurate as Spice. We have formulated the SVM macro-model for 2-stage op-amp and cascode op amp using multi-variate regression using efficient kernel functions for SVM. Multi-objective sizing provides much desired Pareto optimal points from the large design space. Input parameters corresponding to these Pareto points in turn provide appropriate sizes of devices. Further work is being carried out for tuning the SVM kernel parameters, which would enhance the optimality of solution.

\section{REFERENCES}

[1] Least Squares Support Vector Machine Matlab/C Toolbox. [Online]. Available: ttp://www.esat.kuleuven.be/sista/lssvmlab

[2] G. Wolfe and R. Vemuri, "Extraction and use of neural network models in automated synthesis of operational amplifiers," IEEE Transactions on Computer-Aided Design of Integrated Circuits and Systems, vol. 22, no. 2, pp. 198-212, Feb. 2003.

[3] R. Harjani and J. Shao, "Feasibility and performance region modeling of analog and digital circuits," Analog Integrated Circuits and Signal Processing, vol. 10, pp. 23-43, 1996.

[4] F. D. Bernardinis, M. I. Jordan, and A. L. Sangiovanni-Vincentelli, "Support vector machines for analog circuit performance representation," in Proceedings of IEEE/ACM Design Automation Conference, 2003, pp. 964-969.

[5] V. Vapnik, The Nature of Statistical Learning Theory. Springer-Verlag, 1995.

[6] J. A. Suykens, T. Gestel, J. Brabenter, B. Moor, and J. Vandewalle, Least Square Support vector Machines. World Scientific Publishing Co. Pte. Ltd, 2002.

[7] B. Scholkopf and A. J. Smola, Learning with kernels. MIT Press, 1999.

[8] M. Ding and R. Vemuri, "A combined feasibility and performance macromodel for analog circuits," in Proceedings of IEEE/ACM Design Automation Conference, 2005, pp. 63-68.

[9] D. Boolchandani, A. Ahmed, and V. Sahula, "Improved support vector machine regression for analog circuits macromodeling using efficient kernel functions," in IEEE International Workshop on Symbolic and Numerical Methods, Modeling and applications to circuit design, 7-8 October 2008, pp. 61-67.

[10] C. Goh and Y.Li, "GA automated design and synthesis of analog circuits with practical constraints," in 2001 Congress on Evolutionary Computation, 2001, pp. 170-177.

[11] M. Barros, J. Guiherme, and N. Horta, "GA-SVM feasibility model and optimization kernel applied to analog ICs design automation," in $17^{\text {th }}$ ACM Great Lake Symposium on VLSI, USA, 2007, pp. 469-472.

[12] H. Graeb, S. Zizala, J. Eckmueller, and K. Antreich, "The sizing rules method for analog integrated circuit design," in Proceedings of IEEE/ACM International Conference on CAD, 2001, pp. 343-349.

[13] B. De Smedt and G. Gielen, "Watson: design space boundary exploration and model generation for analog and RFIC design," IEEE Transactions on Computer-Aided Design of Integrated Circuits and Systems, vol. 22, no. 2, pp. 213-224, Feb. 2003. 